\title{
Lohnzurückhaltung und Lohnmilitanz: Belgien, Deutschland und die Niederlande, 1950-2010
}

Die Lohnentwicklung entwickelter kapitalistischer Länder weist sowohl historisch gesehen als auch im Ländervergleich erhebliche Unterschiede auf. Erklärungsbedürftig erscheinen vor allem die Schwankungen im Ausmaß der Lohnzuwächse. Ein in der bisherigen Diskussion weitverbreiteter Ansatz besagt, dass starke Gewerkschaften und zentralisierte Lohnfindungssysteme ein vergleichsweise hohes Maß an Lohnzurückhaltung hervorrufen. Anhand einer vergleichenden Untersuchung der Lohnentwicklung in Deutschland, Belgien und den Niederlanden zwischen 1950 und 2010 zeigt der Beitrag jedoch, dass die Lohnzuwächse seit den 1980er Jahren deutlich geringer ausfielen als während der Nachkriegszeit - ein Umstand, der gewerkschaftliche Macht eher als Treiber von Lohnzuwächsen erscheinen lässt. ${ }^{1}$

ERIK BENGTSSON

\section{Einleitung}

Lohnzurückhaltung erscheint vielen Ökonomen als etwas, das den Investitionen und dem Wirtschaftswachstum förderlich ist und Arbeitnehmern wie Arbeitgebern nützt, indem es das Investitionsklima verbessert und die Schaffung von Arbeitsplätzen fördert. Gestützt auf die nachfolgend präsentierten Untersuchungsergebnisse argumentiere ich in diesem Artikel, dass Lohnzurückhaltung weitaus problematischer ist als häufig behauptet. Bei näherem Hinsehen erscheint die Beziehung geringer oder gar nicht vorhandener Lohnzuwächse zu Investitionen weniger evident als ihre Auswirkungen auf die soziale Ungleichheit: Indem sie zu einer einseitigen Bereicherung von Kapitalbesitzern beiträgt, verschärft Lohnzurückhaltung die bestehende Einkommensungleichheit. Von der häufig behaupteten Win-win-Situation kann daher keine Rede sein.

In enger Verbindung mit der Behauptung, dass Lohnzurückhaltung sich positiv auf die wirtschaftliche Dynamik auswirkt, steht die Ansicht, dass starke Gewerkschaften und zentralisierte Lohnverhandlungssysteme die Lohnzurückhaltung begünstigen. Aus dieser Überlegung heraus gelten diese beiden Faktoren als wichtige Voraussetzungen für den wirtschaftlichen Erfolg der Nachkriegszeit. Diese Auffassung wird durch die im Folgenden präsentierte Untersuchung zur Lohnentwicklung in Deutschland, Belgien und den Niederlanden, die den
Zeitraum von 1950 bis 2010 umfasst, auf ihren Wahrheitsgehalt überprüft. Abschnitt 2 gibt einen knappen Überblick über den Forschungsstand und die drei untersuchten Länder. Der hier näher erläuterten gängigen Betrachtungsweise stelle ich einen machttheoretischen Ansatz gegenüber, der starke Gewerkschaften und zentralisierte Lohnverhandlungen nicht als Ursachen der Lohnzurückhaltung, sondern, im Gegenteil hierzu, als Treiber von Lohnzuwächsen ansieht. Nach einer Erläuterung der verwendeten Methoden und Daten (3) werden im darauffolgenden Abschnitt die Ergebnisse der empirischen Analyse präsentiert (4). Dabei werden sowohl Befunde zum historischen Timing der Lohnzurückhaltung (4.1) als auch die Ergebnisse einer Regressionsanalyse zu den Determinanten der Lohnzurückhaltung präsentiert (4.2). Das abschließende Fazit fasst die Resultate zusammen und diskutiert ihre Implikationen für die Forschung (5).

Der Verfasser dankt zwei anonymen Gutachtern der WSI Mitteilungen sowie Philipp Klages in der Redaktion für konstruktive Kritik. Für eventuelle Fehler und Missverständnisse bin nur ich verantwortlich

Übersetzung aus dem Englischen: Klaus Sticker. 


\section{Forschungsstand: Lohnzurückhaltung unter zweierlei Blickwinkel}

\subsection{Betrachtungsweisen der Lohnzurückhaltung}

In der Forschungsliteratur wird überwiegend die Ansicht vertreten, das starke Wirtschaftswachstum in den 1950er und 1960er Jahren sei durch die Kooperation zwischen Arbeit und Kapital ermöglicht worden. Auf der einen Seite hätten sich Arbeiterschaft und Gewerkschaften bei den Lohnforderungen zurückgehalten. Im Gegenzug hierzu hätten die Vertreter der Kapitalseite sich bereit erklärt, die dadurch bedingten Gewinnsteigerungen zu reinvestieren und damit Wachstum und Arbeitsplätze zu schaffen (siehe Eichengreen/Iversen 1999; Eichengreen 2007). In der Regel wird dabei (zumeist implizit) angenommen, dass Lohnzurückhaltung das Wachstum steigere und hierdurch eine Win-win-Situation schaffe. Dieser theoretische Ansatz zur Lohnfindung blendet die Verteilung zwischen Beschäftigten und Kapitalbesitzern aus. Lohnzurückhaltung zu erreichen wird daher als Problem kollektiven Handelns betrachtet, das beide Seiten zu lösen bestrebt sind. Die Frage besteht dieser Auffassung zufolge im Kern lediglich darin, ob die Vertreter von Arbeit und Kapital einander vertrauen und kooperieren (vgl. Lange/Garrett 1985; Wallerstein 1990; siehe Piketty 2014 zur Problematik der Vernachlässigung von Verteilungskämpfen). Vertrauen und Kooperationsbereitschaft, so wird weiter angenommen, würden durch zentrale Lohnverhandlungen gefördert, wodurch die Verhandlungseinheiten „umfassender“ werden und weniger in der Lage sind, die Kosten militanter Lohnpolitik zu externalisieren. Von zentralisierten Lohnverhandlungssystemen glaubt man daher, dass sie Lohnzurückhaltung ermöglichen und hierdurch eine Win-win-Situation hervorrufen.

In den klassischen Studien zur Lohnzurückhaltung (etwa Lange/Garrett 1985; Hicks 1988; Alvarez et al. 1991; Scruggs 2001; Kenworthy 2002) werden Lohnzurückhaltung und Klassenkooperation zwar theoretisch behandelt, eine empirische Erfassung von Lohnentwicklung oder Lohnzurückhaltung fehlt jedoch. Stattdessen wird das BIPWachstum (oder andere Kenngrößen der Wirtschaftsleistung wie Arbeitslosigkeit) mit zentralisierter Lohnfindung korreliert - und einfach angenommen, dass eine positive Korrelation der beiden Variablen bedeutet, eine starke Zentralisierung habe die (nicht erfasste) Lohnzurückhaltung ermöglicht und hierdurch für Wachstum und/oder Beschäftigung gesorgt. Dies ist hoch problematisch, denn neben der Lohnfindung werden BIP-Wachstum und Arbeitslosigkeit von vielen anderen Faktoren beeinflusst. Wer also niedrige Arbeitslosigkeit oder positives Wachstum der Lohnzurückhaltung zuschreibt, ohne die Löhne zu untersuchen, läuft Gefahr, zwar theoretisch komfortable, aber empirisch nicht haltbare Aussagen zur Lohnfindung zu reproduzieren.
Anders als bei diesem koordinationsbezogenen Blickwinkel, unter dem Lohnzurückhaltung Beschäftigen wie Unternehmern gleichermaßen nützt, nehme ich eine Perspektive ein, die von Klassenkonflikt und Machtverhältnissen ausgeht. Dieser Auffassung zufolge fördern starke Gewerkschaften und zentralisierte Lohnverhandlungen, wie in der Nachkriegszeit, eine Umverteilung von Kapital zu Arbeit. In den 1980er Jahren wurde Lohnzurückhaltung in dem Maße wahrscheinlicher, in dem die Gewerkschaften geschwächt wurden, der globale Wettbewerb zunahm und die Arbeitslosigkeit stieg (vgl. Korpi 1983, 2002; Bengtsson 2015). Walter Korpi (2002) hat am umfassendsten untersucht, wie niedrige Arbeitslosigkeit in der Nachkriegszeit mit einer starken Verhandlungsposition der Beschäftigten einherging und umgekehrt hohe Arbeitslosigkeit nach 1980 wesentlich zu einer schwachen Verhandlungsposition beitrug. Die nach 1980 sowohl in den entwickelten kapitalistischen Ländern als auch weltweit im Zusammenhang mit globalen Kapitalströmen beobachtbare Rückkehr der ,industriellen Reservearmee“ bedeutet unter dieser Perspektive, dass die Beschäftigten in ihren Möglichkeiten zu hohen Lohnabschlüssen stärker eingeschränkt sind als zuvor. Unter dem Blickwinkel der Machtverhältnisse fördert eine zentralisierte Lohnfindung zudem nicht nur die Koordinierung, sondern auch die Verhandlungsmacht der Beschäftigten gegenüber den Unternehmern (Ochel 2005, S. 105). Gegenüber der koordinationszentrierten Betrachtungsweise zieht die machtbezogene Perspektive somit die gegenteiligen Hypothesen nach sich: Sie lässt uns weniger Lohnzurückhaltung in den durch stärker zentralisierte Tarifverhandlungen gekennzeichneten Jahren und stärkere Lohnzurückhaltung in der „,neoliberalen Phase“ nach 1980 erwarten.

Der koordinationsbezogene Ansatz mit seinem Schwerpunkt auf Lohnzurückhaltung in der Nachkriegszeit konzentriert sich vor allem auf Länder mit starken oder mittelstarken Gewerkschaften und einem mittleren oder hohen Maß an Verhandlungszentralisierung: insbesondere Kontinentaleuropa und Skandinavien (siehe Eichengreen 2007). Kürzlich wurde für Großbritannien (Hatton/Boyer 2005) sowie Dänemark, Norwegen und Schweden (Bengtsson 2015) gezeigt, dass es in diesen Ländern in der Nachkriegszeit kaum oder keine Lohnzurückhaltung gab, mehr jedoch nach 1980. Gestützt auf Crouchs (1995) Unterscheidung zwischen „,arbeitnehmerdominiertem Korporatismus“ in Skandinavien und „unternehmerdominiertem Korporatismus“ in Kontinentaleuropa weist Bengtsson (2015, im Erscheinen) darauf hin, dass von Lohnzurückhaltung nach dem Krieg eher in Kontinentaleuropa als in Skandinavien die Rede sein kann. Auf dieser Basis werden die hier gegenübergestellten Ansätze zur Analyse der Lohnzurückhaltung nachfolgend mit Blick auf drei wichtige kontinentale Volkswirtschaften untersucht: Belgien, Deutschland und die Niederlande. Dabei werden gegensätzliche Hypothesen (a) zum historischen Timing der Lohnzurückhaltung und (b) zur Art ihrer 
Beziehung zu zentralisierten Lohnverhandlungen überprüft.

\subsection{Die drei Länder}

(West-)Deutschland gilt als der prototypische Fall der Lohnzurückhaltung nach dem Kriege. Beispielsweise stellt Maier (1981, S. 345) fest, dass im Deutschland der späten 1940er Jahre Einigkeit darin herrschte, Konflikte über den jeweiligen Anteil am Nationaleinkommen zugunsten der Früchte künftigen Wirtschaftswachstums aufzuschieben; des Weiteren habe diese Art Einigkeit in der gesamten Nachkriegszeit bestanden. Ähnlich behauptet Kreile (1977, S. 777), die Gewerkschaften seien inflationsavers und um die Ausfuhrleistung besorgt gewesen - der Klassenkonflikt sei zugunsten der Wachstumsdividende ausgesetzt worden. In der Literatur herrscht also eher Konsens über Lohnzurückhaltung im Deutschland der 1950er und 1960er Jahre, wenngleich die Erklärungen zwischen Gewerkschaftsschwäche und Gewerkschaftsideologie schwanken (Carlin 1996, S. 467ff.; Giersch et al. 1993).

Ganz ähnlich wie Deutschland sind die Niederlande für ihre Strategien der Lohnzurückhaltung bekannt. Becker und Hendriks (2008, S. 827) leiten einen Artikel mit der Feststellung ein, Lohnzurückhaltung sei ,the paradigm in the Dutch political economy“. Wirtschaftshistoriker ebenso wie Politologen betonen, dass Lohnzurückhaltung in den 1950er, weniger in den 1960er Jahren, vorherrschte und dann wieder in den frühen 1980er Jahren - seitdem dauerhaft (van Zanden 2000; Visser 1998).

Zwar ist Belgien nach dem Krieg laut Crouch (1995) als Fall eines „unternehmerdominierten Korporatismus“ einzustufen und Gegenstand von Lohnzurückhaltungsanalysen wie der von Eichengreen (2007), sodass wir für diese Zeit dort Lohnzurückhaltung erwarten könnten. Es handelt sich jedoch um einen weniger klassischen Fall als Deutschland und die Niederlande. In den 1950er Jahren waren Lohnverhandlungen nicht im selben Maße tripartistisch und weniger zentralisiert, auch waren weniger zentrale lohnpolitische Ziele (wie Lohnkompression oder Lohnzurückhaltung) gesetzt als in den Niederlanden (De Jong/Soete 1997, S. 18). Analysen der Lohnzurückhaltung in Belgien konzentrieren sich eher auf die 1980er Jahre, also die Zeit nach den turbulenten 1970ern (Cassiers et al. 1996).

Insgesamt kann festgehalten werden, dass sich diese drei Länder als Beispiele eines „unternehmerdominierten Korporatismus“ (Crouch 1995) eignen, um Lohnzurückhaltung und die Erklärungskraft der in der Literatur überwiegenden koordinierungsbezogenen Perspektive zu untersuchen.

\section{Methode und Daten}

\subsection{Methode}

Bei der Lohnzurückhaltung geht es darum, das Reallohnwachstum (also Nominallohnzuwächse abzüglich Inflation) in Bezug auf den im Wesentlichen vom Produktivitätswachstum bestimmten Verteilungsspielraum zu zügeln. Dann lässt sich Lohnzurückhaltung anhand folgender Gleichung messen (vgl. Johnston/Hancké 2009):

Gleichung 1: Lohnzurückhaltung = Reallohnwachstum-Produktivitätswachstum

Ergeben beide Seiten 0, dann gibt es gewissermaßen weder Lohnzurückhaltung (Lohnsteigerung unter der Inflation + Produktivität) noch Lohnmilitanz (Lohnzuwachs über der Inflation + Produktivität). Wegen nicht absehbarer Konjunkturschwankungen sollte die Beziehung für mindestens drei bis vier Jahre Bestand haben, um als signifikantes Muster gelten zu können. Dies wird hier die vorrangige Methode sein, um die aus dem koordinierungsbezogenen und dem machtbezogenen Ansatz hervorgehenden Erwartungen zum historischen Timing der Lohnzurückhaltung seit $1950 \mathrm{zu}$ untersuchen.

Die hier vorgenommene Operationalisierung verwendet Reallohn-, nicht Nominallohnzuwächse. In der Realität werden Nominallöhne festgesetzt, nicht Reallöhne. ${ }^{2}$ Dennoch bleibt der Reallohn das relevante Ergebnis, was (a) Einkommen und Lebensstandard und (b) die Lohnkosten der Unternehmen angeht. Eine eher auf die Absichten bei der Lohnfestsetzung und den Kontext der Aktionen gerichtete Analyse müsste ferner die Inflation gesondert berücksichtigen, doch halte ich es für meine Zwecke - Konzentration auf die Ergebnisse, nicht auf die Absichten der Lohnverhandlungen - für ausreichend, den Reallohn zu betrachten.

Um die entgegengesetzten Ansichten über die Beziehung der Verhandlungszentralisierung zur Lohnzurückhaltung zu untersuchen, werden Regressionsanalysen verwendet.

Eine andere Möglichkeit, Lohnzurückhaltung zu untersuchen, ist die Analyse der Lohnquote, also des Anteils der Arbeitseinkommen am Nationaleinkommen. Werden Lohnzuwächse im Verhältnis zur Produktivität gebremst, sinkt die Lohnquote; umgekehrt steigt sie, wenn die Löhne schneller anwachsen als die Produktivität. Die Lohnquoten sollten dann dem Ergebnis von Gleichung 1 entsprechen. Da sich die hier verwendeten Lohn- und Produktivitätsdaten nur auf den Fertigungssektor beziehen, habe ich die Lohnquoten für die Gesamtwirtschaft

2 Ich danke einem anonymen Gutachter für den Anstoß zur Entwicklung dieses Gedankens. 
einbezogen, um sicherzustellen, dass die Ergebnisse in Bezug auf die hier behandelte Frage kein falsches Bild vermitteln. Die Lohnquoten für die drei Länder (1960 bis 2010) in der AMECO-Datenbank der Europäischen Kommission entsprechen den hier dargelegten Ergebnissen. Gleiches gilt für die Lohnquoten der 1950er Jahre aus den jeweiligen nationalen Quellen (Statistics Netherlands 2011; Statistisches Bundesamt 2012).

\subsection{Daten}

In diesem Artikel werden Reallohn- und Produktivitätsdaten eines internationalen Datensatzes vom US-Bureau of Labor Statistics (BLS 2012) verwendet, in dem Informationen zu Arbeitnehmerentgelten, den geleisteten Arbeitsstunden, der Wirtschaftsleistung sowie der Produktivität des Fertigungssektors enthalten sind. Die Arbeitnehmerentgelte umfassen nicht nur Lohnzahlungen, sondern auch die Arbeitgeberleistungen für Versicherungen und Ähnliches. Dass die Gesamtentgelte die Variable darstellen nicht nur die Löhne -, ist ein Vorzug, da eine Betrachtung lediglich der Löhne und eine Missachtung der nicht lohnbezogenen Leistungen ein falsches Bild des Wohlergehens der Beschäftigten und der Verteilung zwischen Kapital und Arbeit zeichnen können. Die Entgeltzuwächse werden durch den Verbraucherpreisanstieg geschmälert. Als Produktivitätsmaß gilt die reale Wirtschaftsleistung pro Arbeitsstunde. $^{3}$

\section{Empirische Analyse}

\subsection{Das Timing der Lohnzurückhaltung}

Abbildung 1 zeigt die Ergebnisse aus Gleichung 1 für die drei Länder in Fünfjahresdurchschnitten: 1950 bis 2010 für Deutschland und die Niederlande, 1960 bis 2010 für Belgien. Tabelle 1 zeigt die Daten mit verschiedenen Durchschnittswerten (nach Teil-Zeiträumen).

Betrachtet man die Durchschnitte der „Blütezeit“ (1950 bis 1972), so war dies in der belgischen und deutschen Fertigung überhaupt keine Zeit der Lohnzurückhaltung, etwas eher schon in den Niederlanden. Dort war dies allerdings in diesem Zeitraum noch weniger der Fall als in den 1980er Jahren. Eine recht starke Lohnzurückhaltung in allen drei Ländern ist dagegen in der „,neoliberalen Phase“ von 1980 bis 2010 festzustellen: Hier stiegen die Löhne in Deutschland um durchschnittlich $1 \%$ weniger als die Produktivität und in Belgien und den Niederlanden um rund $2 \%$ weniger. Die Daten stützen die machtbezogene Hypothese zur Lohnzurückhaltung nach 1980, die infolge der Schwächung von Gewerkschaften, der Globalisierung und wachsender Arbeitslosigkeit eine stärker ausgeprägte Lohnzurückhaltung
ABB. 1

Zuwächse der Reallöhne abzüglich Produktivitätswachstum, 1950 - 2010

Angaben in Prozent, Fünfjahresdurchschnitte

- Deutschland - Niederlande - Belgien*

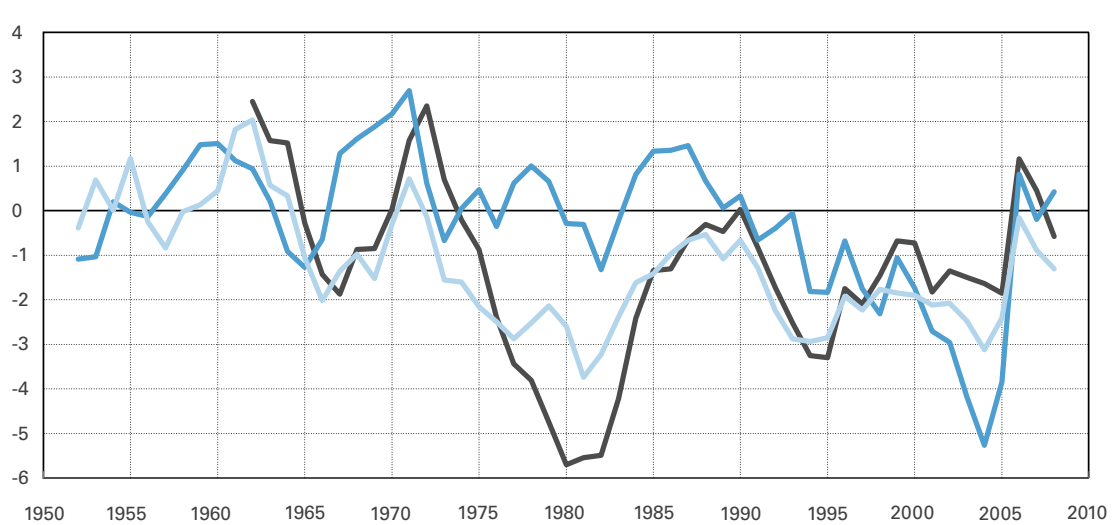

* Werte 1950 und 1955 nicht verfügbar.

Quelle: Bureau of Labour Statistics (2012); Berechnung und Darstellung des Autors.

erwartet. Allerdings gibt es in diesen langen Zeiträumen beträchtliche Schwankungen, die wir im Folgenden diskutieren.

Für Belgien in den 1950er Jahren liegen mir keine Lohndaten vor. Für Deutschland und die Niederlande hingegen, wo die Daten vorhanden sind, lässt sich für die frühen 1950er Jahre eine Lohnzurückhaltung feststellen. Diese Beobachtung befindet sich im Einklang mit dem beispielsweise von Maier (1981) und Giersch et al. (1993) hervorgehobenen Wiederaufbau der Volkswirtschaften. Ähnlich

3 Um die Genauigkeit der Daten zu überprüfen, habe ich die BLS-Schätzungen mit anderen verfügbaren Quellen verglichen. Bei den Produktivitätsdaten für Deutschland habe ich mit Schätzungen des Sachverständigenrats zur Begutachtung der gesamtwirtschaftlichen Entwicklung (Sachverständigenrat 1976, 1998) für den Zeitraum 1950-1991 sowie für den Zeitraum 1970-2010 mit OECD (o. J.) („BIP pro Arbeitsstunde") die Gegenprobe angestellt. Insgesamt ergibt sich ein ähnliches Bild. Insbesondere sind die Trends gleich, doch sind die BLS-Schätzungen variabler, was zu erwarten ist, wenn man, wie beim OECD-Vergleich, eine Schätzung für die Fertigung einer für die Gesamtwirtschaft gegenüberstellt. Die Korrelation zwischen Sachverständigenrat und BLS ist 0.69, die zwischen BLS und OECD ist 0.73. Für Belgien beträgt die BLS-OECD-Korrelation 0.69, die zwischen BLS und De Jong and Soete (1997) für 1960-1989 ebenfalls 0.69. Für die Niederlande ist die BLS-OECD-Korrelation 0.72, die zwischen BLS und De Jong and Soete (1997) 0.70. Insgesamt scheinen die BLSSchätzungen vertrauenswürdig: Sie ergeben die gleichen Trends wie die anderen Schätzungen, jedoch variablere Ergebnisse als für die Gesamtwirtschaft. 
TABELLE 1

\section{Zuwächse der Reallöhne abzüglich Produktivitätswachstum, Durchschnitte pro Zeitabschnitt}

Angaben in Prozent

\begin{tabular}{|c|c|c|c|c|c|c|c|c|c|c|c|c|}
\hline & $1950-54$ & $1955-59$ & $1960-64$ & $1965-69$ & $1970-74$ & $1975-79$ & $1980-84$ & $1985-89$ & $1990-94$ & 1995-99 & 2000-04 & $2005-10$ \\
\hline Belgien & nicht verfügbar & nicht verfügbar & 2.45 & -1.88 & 2.35 & -3.44 & -5.49 & -0.66 & -1.73 & -2.10 & -1.36 & -0.35 \\
\hline Deutschland & -1.09 & 0.38 & 0.94 & 1.28 & 0.61 & 0.62 & -1.33 & 1.46 & -0.39 & -1.75 & -2.96 & -0.93 \\
\hline Niederlande & -0.39 & -0.84 & 2.04 & -1.36 & -0.14 & -2.88 & -3.23 & -0.66 & -2.24 & -2.23 & -2.08 & -1.46 \\
\hline
\end{tabular}

\begin{tabular}{|c|c|c|c|}
\hline & „Blütezeit“ 1950-1972 & „Neoliberale Phase“ 1980-2010 & $1950-2010$ \\
\hline Belgien & 0.57 & -1.90 & -1.20 \\
\hline Deutschland & 0.34 & -0.98 & -0.28 \\
\hline Niederlande & -0.14 & -1.97 & -1.29 \\
\hline
\end{tabular}

sieht Bengtsson (2015) Lohnzurückhaltung Anfang der 1950er Jahre in Dänemark und Norwegen, in zwei Ländern also, die im Zweiten Weltkrieg besetzt waren und noch um 1950 mit dem Wiederaufbau zu ringen hatten. Anders verhielt es sich im nicht besetzten Schweden, das seine Wirtschaft nicht neu aufbauen musste. Im Deutschland der zweiten Hälfte der 1950er Jahre begannen die Löhne schneller zu steigen als die Produktivität; diese Tendenz verstärkt sich - nicht überraschend - von 1965 bis 1969, den Jahren der berühmten Lohnexplosion in Europa. Auch die Niederlande verzeichnen um 1960 einen dramatischen Wandel und den Übergang in eine Phase, in der die Löhne rascher als die Produktivität wachsen, wie von Visser (1998) und van Zanden (2000) beschrieben.

In allen drei Ländern herrscht Anfang der 1980er Jahre Lohnzurückhaltung, in Belgien und den Niederlanden mehr noch als in Deutschland. Der Wechsel von Lohnmilitanz in den 1970er Jahren zur Lohnzurückhaltung in den 1980er Jahren erweist sich in Belgien als besonders radikal; diese deutliche Schwankungsanfälligkeit mag darauf zurückzuführen sein, dass dort die Lohnfindung weniger koordiniert als in Deutschland und weniger zentralisiert als in den Niederlanden war, oder möglicherweise auch auf die eher unregelmäßige industrielle Entwicklung in dieser Phase (vgl. Cassiers et al. 1996). Umgekehrt zeigt sich in Deutschland - ebenfalls nicht überraschend - eine besonders starke Lohnzurückhaltung in den 1990er und 2000er Jahren (vgl. Bispinck 2011). Vor diesem Hintergrund ist es folgerichtig, dass Erwerbsarmut und Ungleichheit Gegenstand aktueller heftiger Kontroversen in Deutschland sind.

\subsection{Zentralisierte Lohnfindung}

Soweit hat sich die empirische Analyse hier auf die zeitliche Dimension konzentriert, gemäß der Unterscheidung zwischen dem Ansatz zur Lohnzurückhaltung in der Nach- kriegszeit (1950er und 1960er Jahre) und dem zur Lohnzurückhaltung in der neoliberalen Phase nach 1980. Als Nächstes wird die Lohnzurückhaltung in Bezug auf die Verhandlungsebenen untersucht, da ein Großteil der ökonomischen Literatur zu diesem Thema die Lohnfindung in Bezug auf den Zentralisierungsaspekt und weniger auf den Zeitabschnitt diskutiert. Diese beiden Bezugsrahmen nähern sich in gewisser Weise an, da Tarifverhandlungen in der Nachkriegszeit im Allgemeinen stärker zentralisiert waren als nach 1980, dennoch bleiben sie unterscheidbar.

Im Folgenden werden die Ergebnisse einer Regressionsanalyse (nach der Methode der kleinsten Quadrate) präsentiert. Als abhängige Variable dient hierbei das Ergebnis der Gleichung 1 (Reallohnwachstum - Produktivitätswachstum). Die zentrale unabhängige Variable ist ein Maß der Verhandlungsebene, entweder nach Golden und Wallerstein (2006, Variable „barglev2“) für die Jahre 1950 bis 2000 oder nach Visser (2013, Variable „level“) für den Zeitraum 1960 bis 2010. Beide werden auf einer Skala von 1 bis 5 abgetragen, auf der "1“ völlig dezentralisierte Tarifverhandlungen (auf Betriebsebene) und ,5“ zentralisierte Verhandlungen auf landesweiter Ebene bedeuten. Mit anderen Worten: Nach der koordinationsbezogenen Sicht auf Lohnzurückhaltung wäre ein negativer und statistisch signifikanter Koeffizient für die Variable „Verhandlungsebene“ zu erwarten. Die Zeit wird statistisch kontrolliert, eine Jahresvariable eingeschlossen; dabei wäre ein linearer negativer Effekt zu erwarten, gemäß der machtbezogenen These zur Lohnzurückhaltung. Außerdem wird Arbeitslosigkeit statistisch kontrolliert, da in der Literatur meist die Auffassung vertreten wird, dass sich Lohnzuwächse bei höherer Arbeitslosigkeit reduzieren. ${ }^{4}$

4 Daten zur Arbeitslosigkeit nach OECD (o. J.), außer Belgien 1950-1959, Deutschland 1950-1955 und Niederlande 1950-1954, siehe hierzu Mitchell (1992). 
TABELLE 2

Einflussfaktoren auf das Ausmaß der Lohnzurückhaltung. Ergebnisse der Regressionsanalysen

\begin{tabular}{lc|c}
\hline (Modell-Nr.) & (1) & (2) \\
\hline \multicolumn{2}{c}{ Aggregiert } \\
\hline Verhandlungsebene & $\begin{array}{c}-0.66^{* *} \\
(0.28)\end{array}$ & $\begin{array}{c}-0.46^{*} \\
(0.28)\end{array}$ \\
\hline$\Delta$ Arbeitslosigkeit & & $-1.03^{* *}$ \\
& & $(0.25)$ \\
\hline Organisationsgrad & & \\
\hline Jahr & $-0.06^{* * *}$ & $\begin{array}{c}-0.05^{* * *} \\
(0.02)\end{array}$ \\
\hline Konstante & $(0.02)$ & $106.46^{* * *}$ \\
\hline Beobachtungen, & $111.42^{* * *}$ & $(34.18)$ \\
\hline Zeitraum & $(35.04)$ & 141, \\
\hline Adjusted R-squared & 0.09 & 0.19 \\
\hline
\end{tabular}

\begin{tabular}{c|c}
\hline (3) & \multicolumn{1}{c}{ (4) } \\
\hline \multicolumn{2}{c}{ Fixed-Effects } \\
\hline-0.32 & -0.10 \\
$(0.34)$ & $(0.33)$ \\
\hline & $\begin{array}{r}1.09 * * * \\
(0.25)\end{array}$ \\
\hline & \\
\hline & \\
\hline 0.41 & -0.42 \\
\hline$(1.26)$ & $(1.21)$ \\
\hline 143, & 141, \\
\hline $1950-2000$ & $1950-2000$ \\
\hline 0.01 & 0.12 \\
\hline
\end{tabular}

\begin{tabular}{ccc}
\hline $\mathbf{( 5 )}$ & $\mathbf{( 6 )}$ & $\mathbf{( 7 )}$ \\
\hline \multicolumn{3}{c}{ Aggregiert } \\
\hline-0.48 & -0.42 & -0.56 \\
$(0.40)$ & $(0.37)$ & $(0.38)$ \\
\hline & $-1.53^{* * *}$ & $-1.62^{* *}$ \\
& $(0.30)$ & $(0.32)$ \\
\hline & & 0.40 \\
& & $0.30)$ \\
\hline $0.06 * * *$ & $-0.06^{* * *}$ & $-0.05^{* * *}$ \\
$(0.02)$ & $(0.02)$ & $(0.02)$ \\
\hline $110.80^{* * *}$ & $124.15^{* * *}$ & $106.65^{* * *}$ \\
$(40.69)$ & $(37.81)$ & $(39.32)$ \\
\hline 153, & 153, & 150 \\
\hline $1960-2010$ & $1960-2010$ & $1960-2010$ \\
\hline 0.05 & 0.19 & 0.18 \\
\hline
\end{tabular}

\begin{tabular}{|c|c|c|}
\hline (8) & (9) & (10) \\
\hline \multicolumn{3}{|c|}{ Fixed-Effects } \\
\hline \multirow[t]{3}{*}{$\begin{array}{l}-0.11 \\
(0.56)\end{array}$} & $\begin{array}{c}0.07 \\
(0.52)\end{array}$ & $\begin{array}{l}-0.14 \\
(0.53)\end{array}$ \\
\hline & $\begin{array}{c}-1.48 * * * \\
(0.31)\end{array}$ & $\begin{array}{c}-1.56^{* * *} \\
(0.33)\end{array}$ \\
\hline & & $\begin{array}{l}0.53^{*} \\
(0.31)\end{array}$ \\
\hline $\begin{array}{l}-0.56 \\
(2.04)\end{array}$ & $\begin{array}{l}-1.12 \\
(1.91)\end{array}$ & $\begin{array}{l}-0.32 \\
(1.92)\end{array}$ \\
\hline $\begin{array}{c}153 \\
1960-2010\end{array}$ & $\begin{array}{c}153 \\
1960-2010\end{array}$ & $\begin{array}{c}150 \\
1960-2010\end{array}$ \\
\hline 0.00 & 0.13 & 0.14 \\
\hline
\end{tabular}

Anmerkung: Regressionen geschätzt nach der Methode der kleinsten Quadrate. Standardfehler in runden Klammern. *** $p<0.01, * * p<0.05,{ }^{*} p<0.1$ Quelle: Berechnungen des Autors.

Wie gezeigt, ist für die machtbezogene Perspektive ferner die hohe Arbeitslosigkeit seit etwa 1980 von großer Bedeutung (Korpi 2002). Des Weiteren wird der gewerkschaftliche Organisationsgrad (nach Daten von Visser 2013) statistisch kontrolliert, wobei ein positiver Effekt für den Mehrbetrag im Verhältnis Löhne-Produktivität zu erwarten ist. Es werden sowohl aggregierte Modelle - in denen alle Länder gemeinsam betrachtet werden - als auch Fixed-Effects-Modelle verwendet, bei denen ausschließlich die Variation im einzelnen Land betrachtet wird. Tests zur Autokorrelation der Zeitreihen und Unit-Root-Tests (Augmented DickeyFuller-Test) der abhängigen Variablen ergeben weder eine Autokorrelation noch Unit-Roots, wobei sowohl Arbeitslosigkeit als auch Organisationsgrad Unit-Roots aufweisen. Die abhängige Variable und die Verhandlungsebene werden dann in Ebenen in die Modelle eingegeben, die Arbeitslosigkeit und der Organisationsgrad in Veränderungen. Tabelle 2 enthält die Regressionsergebnisse.

Bei neun von zehn Modellen ist der Koeffizient für die Verhandlungsebene negativ. Allerdings ist er nur bei zwei Modellen (aggregierte Regressionen mit der Stichprobe 1950 - 2000) statistisch signifikant. Durch die Kontrolle der Länder-Heterogenität oder der Arbeitslosigkeit wird der Koeffizient der Verhandlungsebene kleiner, auch durch die Verwendung der Stichprobe 1960 - 2010. Somit stützen unsere Ergebnisse insgesamt nicht die These, zentralisierte Tarifverhandlungen seien mit größerer Lohnzurückhaltung in diesen drei Ländern verbunden. Die Ergebnisse für den gewerkschaftlichen Organisationsgrad, wo wir unter der Machtperspektive einen positiven Effekt erwarten würden, sind allerdings gleichermaßen schwach: Bei beiden Model- len mit dieser Variable ist der Koeffizient zwar erwartungsgemäß positiv ( 0.40 und 0.53 ), aber nur in einem Modell statistisch signifikant. Andererseits ist der Effekt der Arbeitslosigkeit, der von Korpi (2002) in seiner Analyse der Verschiebung der Machtverhältnisse von Beschäftigten auf Unternehmer hervorgehoben wird, sehr stark und durchgängig signifikant. Der Effekt der Zeitvariablen stellt sich ebenfalls so dar, wie unter der Machtperspektive zu erwarten ist: Lohnzurückhaltung ist gegen Ende des Abschnitts 1950 - 2010 weiter verbreitet als zu Beginn. Insgesamt bestätigen die Regressionsergebnisse nicht die These, eine zentralisierte Lohnfindung fördere die Lohnzurückhaltung. Hingegen zeigt das stärkste Ergebnis erneut, dass Lohnzurückhaltung eher ein Phänomen der späteren als der früheren Phase des untersuchten Zeitraums ist.

\section{Fazit}

Insgesamt wird die koordinationsbezogene Sicht auf Lohnzurückhaltung, die in der Forschung zum Thema vorherrscht, durch die hier präsentierten Ergebnisse nicht bestätigt. Anders als in den Erklärungen der Nachkriegswirtschaftsleistung - etwa Eichengreen und Iversen (1999) und Eichengreen (2007) -, jedoch im Einklang mit der jüngsten Kritik (Hatton/Boyer 2005; Bengtsson 2015) hat die Untersuchung gezeigt, dass es in den 1950er und 1960er Jahren kaum, nach 1980 jedoch beträchtliche Lohnzurückhaltung gab. Dies entspricht dem, was Korpi (2002) 
erwarten lässt: Dieser betont, wie die Massenarbeitslosigkeit seit Beginn der 1980er Jahre den Handlungsspielraum der Beschäftigten in den entwickelten kapitalistischen Ländern einschränkt. Der unter anderem von Scruggs (2001) und Kenworthy (2002) behauptete Zusammenhang zwischen zentralisierter Lohnfindung und Lohnzurückhaltung erweist sich demgegenüber als zweifelhaft. Aus der Sicht Bengtssons (2015), wonach Lohnzurückhaltung in der Nachkriegszeit eher im „liberalen Korporatismus“ Belgiens, der Niederlande und Deutschlands als im „sozialen Korporatismus" der skandinavischen Länder zu finden sei, überraschen die hier beschriebenen Ergebnisse. Eine wichtige Aufgabe für die weitere Forschung wäre, die empirische Untersuchung auf weitere relevante Länder wie Finnland und Österreich auszuweiten, um mehr Erkenntnisse zur Lohnzurückhaltung zu gewinnen. Eine weitere Vertiefung könnte eine Studie leisten, die sich den Wechselwirkungen zwischen Lohnfindung, Geldpolitik, Wechselkursen und Verteilungsergebnissen widmet, wie im Abschnitt zur Methode beschrieben.

Im Einzelnen ergeben sich drei Konsequenzen für den fachlichen Diskurs: Auf der analytischen Ebene hat sich die koordinationsbezogene Sichtweise auf Lohnfindung, wie sie von Garrett und Lange (1989); Hicks (1988); Kenworthy (2002) und Scruggs (2001) vertreten wird, als empirisch nicht stichhaltig erwiesen. Das grundlegende Problem von Analysen dieser Art besteht darin, dass die ihnen zugrunde liegenden Überlegungen die Existenz von Klassenkonflikten nicht hinreichend berücksichtigen. Mit der Annahme, Lohnzurückhaltung schaffe Arbeitsplätze und Lohnmilitanz fördere die Arbeitslosigkeit, blendet der vorherrschende Ansatz die Verteilungskämpfe zwischen Kapital und Arbeit aus und vermittelt ein falsches Bild von der Lohnfindung und ihrer Bedeutung für die Wirtschaft. Tatsächlich können Lohnquoten steigen, ohne zu höherer Arbeitslosigkeit zu führen (vgl. Piketty 2014). Zudem werden Lohnabschlüsse von der relativen Verhandlungsstärke der beiden Seiten beeinflusst. Lohnfindung und Lohnzurückhaltung sollten daher von der politischen Ökonomie nicht nur mit Blick auf die Struktur der Lohnverhandlungssysteme, sondern auch im Hinblick auf die mit diesen einhergehenden Machtverhältnisse untersucht werden (vgl. Streeck 2011).

Der zweite Punkt betrifft die Beziehung zwischen Lohnzurückhaltung und Investitionen. Dass es in der Nachkriegszeit starkes Wachstum ohne viel Lohnzurückhaltung gab, stellt die verbreitete, als zwangsläufig dargestellte Verknüpfung von Lohnzurückhaltung mit einem hohen Investitionsvolumen infrage. In der Nachkriegszeit traten die sinkende Rentabilität, fortgesetzte Investitionen und starkes Wirtschaftswachstum gemeinsam auf (vgl. Piketty 2014). Seitdem hat sich die Beziehung zwischen Löhnen einerseits und Arbeitslosigkeit, Investitionen und Wachstum andererseits offenbar verändert: eine Transformation, die für die politische Ökonomie von großem Interesse sein sollte. Wie Baccaro/Benassi (2013) feststellen, hält die Vergleichende politische Ökonomie sehr an der gängigen These der 1980er Jahre fest, dass Lohnzurückhaltung stets zu mehr Beschäftigung führe. Ignoriert wird dabei die Beziehung zwischen Löhnen und Nachfrage: Die Nachfrage nach Gütern und Dienstleistungen speist sich weitgehend aus Löhnen, sodass sich geringe Lohnzuwächse negativ auf die Nachfrage auswirken und hierdurch das Wachstum beeinträchtigen können. Die Beziehung zwischen Löhnen und Wachstum gestaltet sich damit offener und entspricht eher den von Vertretern des Postkeynesianismus entwickelten Modellen (z. B. Stockhammer 2011) oder der im Rahmen der deutschen lohnpolitischen Debatte der 1960er und 1970er Jahre vertretenen Sichtweise (Roth 1982). Politische Ökonomen sollten die Beziehungen zwischen Löhnen und Investitionen, Wachstum und Arbeitslosigkeit rekonzeptuali- sieren und dabei weniger deterministisch vorgehen. Hierbei sollte nicht nur die Möglichkeit berücksichtigt werden, dass sich die Art der postulierten Wirkungsbeziehungen im Zeitverlauf verändern können, sondern auch nach Erklärungen für solche Veränderungen gesucht werden.

Und drittens: Die Debatte über Lohnzurückhaltung wäre zusammen mit der Debatte über Ungleichheit zu führen. Das Kapitalvermögen konzentriert sich immer mehr bei den Spitzenverdienern, der Abstand zu den Arbeitseinkommen wird größer; dazu stellt Piketty (2014, S. 323) fest: „Die Ungleichheiten hinsichtlich des Kapitals (...) sind (...) stets extreme Ungleichheiten“. Wie Marx (2007) in einer Studie zu den Niederlanden darlegt, wirkt sich Lohnzurückhaltung in Richtung einer Absenkung der Lohnquote (bei gleichzeitigem Anstieg der Kapitalquote) aus und trägt damit entscheidend zur Vergrößerung der Einkommensungleichheit bei. In jüngerer Zeit zeigen Untersuchungen zu den USA, wie sich das Wirtschaftswachstum von der Lohnentwicklung abgekoppelt hat. Deutlich werden dabei auch die im Lohnrückgang nach 1980 zum Ausdruck kommende Veränderung der Machtverhältnisse sowie das Anwachsen von Ungleichheit und Armut (Bailey et al. 2011). Lohnzurückhaltung ist eben doch etwas anderes als eine unproblematische Win-win-Situation.

\section{LITERATUR}

Alvarez, R. M./Jarrett, G./Lange, P. (1991): Government partisanship, labor organization, and macroeconomic performance, in: American Political Science Review 85 (2), S. $539-556$

Baccaro, L./Benassi, C. (2013): Softening industrial relations institutions, har dening growth model:The transformation of the German political economy, Papier zur 20 $0^{\text {th }}$ Conference of Europeanists, Amsterdam, June 2013

Bailey, J./Coward, J./Whittaker, M. (2011): Painful separation: An international study of the weakening relationship between economic growth and the pay of ordinary workers, London

Becker, U./Hendriks, C. (2008): As the central planning bureau says. The Dutch wage restraint paradigm, its sustaining epistemic community and its relevance for comparative research, in: Review of International Political Economy 15 (5), S. $826-850$

Bengtsson, E. (2015): Wage restraint in Scandinavia:The postwar period or the neoliberal age?, in: European Review of Economic History (im Erscheinen)

Bispinck, R. (2011): Pay in the 2000s: Development and outcomes, WSI Report 06, Düsseldorf

Bureau of Labor Statistics (2012): International comparisons of manufacturing productivity and unit labor cost trends, 2011, Washington DC, http://www.bls. gov/ilc/\#productivity (letzter Zugriff am 07. 08. 2014)

Carlin, W. (1996): West German growth and institutions, 1945-90, in: Crafts,

N./Toniolo, G. (Hrsg.): Economic growth in Europe since 1945, Cambridge, S. $455-497$

Cassiers, I./De Villé, P./Solar, P. M. (1996): Economic growth in postwar Belgium, in: Crafts, N./Toniolo, G. (Hrsg.): Economic growth in Europe since 1945, Cambridge, S. 173-209

Crouch, C. (1995): Conclusion: Reconstructing corporatism? Organized decentralization and other paradoxes, in: Crouch, C./Traxler, F. (Hrsg.): Organized industrial relations in Europe: What future?, Aldershot, S. 311-333

De Jong, H./Soete, A. (1997): Comparative productivity and structural change in Belgian and Dutch manufacturing, 1936-1987, Research Memorandum GD-36, Groningen

Eichengreen, B. (2007): The European economy since 1945: Coordinated capitalism and beyond, Princeton

Eichengreen, B./Iversen, T. (1999): Institutions and economic performance: Evidence from the labour market, in: Oxford Review of Economic Policy 15 (4), S. $121-138$

Garrett, G./Lange, P. (1989): Government partisanship and economic performance, in: Journal of Politics 51 (3), S. 676-693

Giersch, H./Paqué, K.-H./Schmieding, H. (1993): Openness, wage restraint, and macroeconomic stability: West Germany's road to prosperity 1948-1959, 
in: Dornbusch, R./Plölling, W./ Layard, R.G. (Hrsg.): Postwar economic reconstruction and lessons for the East today, Cambridge, S. 1-27

Golden, M./Wallerstein, M. (2006): Union centralization among advanced industrial societies: Update to 1995/2000, http://thedata.harvard.edu/dvn/dv/ goldenm (zuletzt aufgerufen am 7. August 2014)

Hatton, T./Boyer, G. (2005): Unemployment and the UK labour market before, during and after the golden age, in: European Review of Economic History 9 (1), S. $35-60$

Hicks, A. (1988): Social democratic corporatism and economic growth, in: Journal of Politics 50 (3), S. 677-704

Johnston, A./Hancké, B. (2009): Wage inflation and labour unions in EMU, in: Journal of European Public Policy 16 (4), S. 601-622

Kenworthy, L. (2002): Corporatism and unemployment in the 1980s and 1990s, in: American Sociological Review 67 (3), S. 367-388

Korpi, W. (1983): The democratic class struggle, London

Korpi, W. (2002): The great trough in unemployment: A long-term view of unemployment, inflation, strikes, and the profit/wage ratio, in: Politics and Society 30 (3), S. 365-426

Kreile, M. (1977): West Germany: The dynamics of expansion, in: International Organization 31 (4), S. 775-808

Lange, P./Garrett, G. (1985): The politics of growth: Strategic interaction and economic performance in the advanced industrial democracies, 1974-1980, in: Journal of Politics 47 (3), S. 792-827

Maier, C. (1981): The two postwar eras and the conditions for stability in twentieth-century Western Europe, in: American Historical Review 86 (2), S. 327-352 Marx, I. (2007): The Dutch 'miracle' revisited: The impact of employment growth on poverty', in: Journal of Social Policy 36 (3), S. 383-397

Mitchell, B. R. (1992): International historical statistics: Europe 1750-1988, London Ochel, W. (2005): Decentralizing wage bargaining in Germany: A way to increase employment?, in: Labour 19 (1), S. 91-121

OECD (o.J.): Productivity, level of GDP per capita and productivity, GDP per hour worked, http://stats.oecd.org/Index.aspx?DataSetCode=PDB_LV (zuletzt aufgerufen am 14. August 2014)

Piketty, T. (2014): Das Kapital im 21. Jahrhundert, München

Roth, J. (1982): Mehr Beschäftigung durch Reallohnzurückhaltung: Zum Streit zwischen kosten- und nachfrageorientierter Lohnpolitik, Kieler Diskussionsbeiträge Nr. 85, Kiel

Sachverständigenrat zur Begutachtung der gesamtwirtschaftlichen Entwicklung (1976): Zeit zum Investieren, Jahresgutachten 1976/77, Stuttgart
Sachverständigenrat zur Begutachtung der gesamtwirtschaftlichen Entwicklung (1998): Vor weitreichenden Entscheidungen, Jahresgutachten 1998/1999, Stuttgart

Scruggs, L. (2001): The politics of growth revisited, in: Journal of Politics 63 (1), S. $120-140$

Statistics Netherlands (2011): National accounts 2010, Den Haag Statistisches Bundesamt (2012): Deutschland Statistisches Jahrbuch 2012, Wiesbaden

Stockhammer, E. (2011): Peripheral Europe's debt and German wages, in: International Journal of Public Policy 7 (1), S. 83-96

Streeck, W. (2011): Taking capitalism seriously: Towards an institutionalist approach to contemporary political economy, in: Socio-Economic Review 9 (1), S. $137-167$

Van Zanden, J. L. (2000): Post-War European economic development as an out of equilibrium growth path: The case of the Netherlands, in: De Economist 148 (4), S. $539-555$

Visser, J. (1998): Two cheers for corporatism, one for the market: industrial relations, wage moderation and job growth in the Netherlands, in: British Journal of Industrial Relations 36 (2), S. 269-292

Visser, J. (2013): Data base on institutional characteristics of trade unions, wage wetting, state intervention and social pacts, 1960-2011 (ICTWSS). http://www.uva-aias.net/208 (zuletzt aufgerufen am 14. August 2014)

Wallerstein, M. (1990): Centralized bargaining and wage restraint, in: American Journal of Political Science 34 (4), S. 982-1004

\section{AUTOR}

ERIK BENGTSSON, PhD, Wissenschaftler an der Universität Göteborg, Department of Economy and Society. Arbeitsschwerpunkte: Lohnfindung, funktionale Einkommensverteilung, Löhne in der Wirtschaftsgeschichte

erik.bengtsson@econhist.gu.se 